\title{
CALIDAD NUTRICIONAL DE LA CENA CONSUMIDA POR ADULTOS MAYORES HOSPEDADOS EN UN HOTEL DEL MUNICIPIO DE BALNEÁRIO CAMBORIÚ, SANTA CATARINA, BRASIL
}

\author{
NUTRITIONAL QUALITY OF THE DINNER CONSUMED \\ BY ELDERLY PEOPLE STAYING IN A HOTEL IN \\ BALNEÁRIO CAMBORIÚ, BRAZIL
}

\author{
Márcia Reis F., Priscilla Vanhoni R. \\ Centro de Ciencias de la Salud. \\ Universidad del Valle del Itajaí - Itajaí, Santa Catarina, Brasil.
}

\begin{abstract}
Objective: To evaluate the nutritional quality of the meals consumed by 105 elderly tourists in a hotel. Method: To ensure the accuracy of the data, three portions of each option were collected and weighed. The three weights were then averaged to create a standard measure. The number of portions that each tourist took for their meal were recorded and later converted to grams using the standard measure. When evaluating the nutritional composition of the selected foods, calories, macronutrients, saturated fat, cholesterol, sodium and fiber content were all taken into consideration. Results: Most of the tourists were females (70\%) from Argentina (88\%) between the ages of 60 and 85 (85\%). It was noted that the males consumed foods with a higher caloric content (175\% of recommended amounts), total fat (235\%), and saturated fat, cholesterol (151\%), proteins (170\%) and sodium (133\%). The quantities of carbohydrates (102\%) and fiber (98\%) were appropriate to meet dietary needs.
\end{abstract}

Key words: Elderly; tourism; dietetic; food consume; hotel.

Este trabajo fue recibido el 25 de Noviembre de 2008 y aceptado para ser publicado el 5 de Mayo de 2009.

\section{INTRODUCCIÓN}

La población mundial está envejeciendo. Los países latinoamericanos están aumentando aceleradamente el número de individuos en edad adulta y avanzada, hecho que puede resultar de la mejoría económica y social de la población (1-3).

En Brasil, según el IBGE (Instituto Brasileño de Geografía y Estadística) en el año 2000, 15,5 millones de habitantes tenían 60 años o más, equivalente al $10 \%$ de la población total. Dicho Instituto estima que en 2025 este número será superior a 33 millones, lo que corresponderá al $14 \%$ de la población brasileña (4).

Con el envejecimiento aumenta la prevalencia de enfermedades, así que es importante el mejor conocimiento de las enfermedades asociadas al aumento de la edad, estado nutricional y cambios corporales, fisiológicos y sociales de los adultos mayores (5).

Los cambios fisiológicos que ocurren durante el envejecimiento pueden interferir en el estado nutricional, ya que las alteraciones en el sistema digestivo, pérdidas sensoriales, problemas de masticación, disminución de la tasa metabólica basal (TMB), reducción de la palatabilidad por gustos primarios como dulce, amargo, salado y ácido pueden llevar al bajo consumo de alimentos por estas personas y, consecuentemente, a la ingestión inadecuada de nutrientes esenciales $(6,7)$.

En esta fase de la vida ocurre una mayor incidencia de enfermedades crónicas no transmisibles como la hipertensión arterial, cáncer, diabetes tipo 2 y enfermedades cardiovasculares, estando estas enfermedades asociadas a restricciones alimentarias y a un menor aprovechamiento de los nutrientes. También el consumo de medicamentos, juntamente con factores psicosociales como la depresión, exclusión social y capacidad de desplazamiento, pueden tener un papel importante en el estado nutricional de estos individuos (8). 
El turismo para la tercera edad, como manifestación cultural y psicosocial, es un importante instrumento de inclusión del adulto mayor, que le permite una participación más efectiva en la sociedad. Sin embargo, su permanencia fuera de casa exige algunos cuidados relacionados con su salud y bienestar (9).

Una investigación reciente verificó que en hoteles de Balneário Camboriú, durante la cena de huéspedes en régimen de media pensión, se ofertaba una gran variedad de alimentos y comidas en buffet de autoservicio, con predominio de las ricas en energía, con alta cantidad de grasas y de carbohidratos refinados (10).

Cuando el adulto mayor está viajando, queda más vulnerable, una vez que factores como el cambio de rutina, las condiciones presentadas por el destino turístico y la curiosidad por vivenciar nuevas experiencias pueden influenciar en su consumo de alimentos y llevarlo a elegir opciones que pueden agravar su estado de salud.

Pese a la importancia del tema, se han realizado pocas investigaciones sobre este tema, existiendo la necesidad de un mayor conocimiento sobre el comportamiento alimentario del adulto mayor cuando está de viaje.

El presente trabajo tuvo como objetivo evaluar la calidad de los alimentos consumidos en la cena por turistas de la tercera edad, hospedados en un hotel del municipio de Balneário Camboriú, estado de Santa Catarina.

\section{SUJETOS Y MÉTODO}

Se seleccionaron 105 individuos de 60 años o más, hospedados en un hotel de Balneário Camboriú y que estaban cenando en el restaurante del hotel en el momento de la recolección de datos. Se calculó esta muestra con base en una prevalencia de inadecuación de consumo del $50 \%$, un margen de error del $10 \%$ y un intervalo de confianza del $95 \%$.

Para la evaluación de la comida consumida, inicialmente se realizó la estandarización del peso medio de los alimentos servidos con los respectivos utensilios utilizados por el restaurante antes de éste estar abierto a los usuarios. Se obtuvieron dos porciones de cada uno de los alimentos, en diferentes medidas: 1leno (a) y raso (a) o unidad grande y pequeña.

Para esta estandarización se realizaron tres pesajes en balanza OHAUS LS2000 con capacidad para 2000 gramos. Se efectuó la evaluación de la calidad nutricional a partir de la observación directa del consumo de alimentos durante la cena. El turista elaboraba el montaje de su plato, sin interferencia de los entrevistadores. Se observó cuidadosamente este proceso y todos los alimentos y porciones servidas y consumidas fueron registradas en un formulario propio.

Con los apuntes obtenidos en la observación directa del montaje de la comida, se realizó el cálculo en gramos del consumo alimentario en la cena.

Para el cálculo nutricional se utilizó el software “Dietwin Profissional Versão 2.0.17". En la evaluación de la composición nutricional de la comida, se analizaron los datos sobre el valor energético, macronutrientes, grasa saturada, colesterol, sodio y fibra.

Se calcularon las necesidades de energía con base en las Dietary Reference Intakes (DRIs, 2002), utilizándose como referencia la edad (años), altura (mt), peso $(\mathrm{kg})$ y nivel de actividad física informados por la población estudiada (11).

Para la evaluación de la calidad nutricional de la cena, se consideró que los individuos deberían consumir en esta comida el $40 \%$ de sus necesidades diarias de energía y nutrientes, con base en el porcentual máximo preconizado para este tipo de comida (12), ya que la mayoría de los adultos mayores hospedados en hoteles de Balneário Camboriú acostumbraba participar de paquetes promocionales que incluían en el valor del hospedaje el desayuno y la cena, siendo, la mayoría de las veces, ésta la única gran comida realizada por ellos durante el día (10).

Se realizó la adecuación de la media de los macronutrientes disponibles para el consumo de la cena a partir de las recomendaciones de la FAO/WHO (2003), definidos como estándares el $55-75 \%$ del total de las necesidades de energía (NET) para carbohidratos, el $10-15 \%$ para proteínas, el $15-30 \%$ para grasas y valores menores o iguales al $10 \%$ para grasa saturada, al máximo $120 \mathrm{mg}$ de colesterol, y una cantidad mínima de 8,4 g de fibras (DRIs, 2002). Para el sodio, se definieron valores inferiores a $920 \mathrm{mg}$, correspondientes al $40 \%$ del nivel máximo de ingestión tolerable (UL) para este mineral con base en las DRIs $(2004)(13,11,14)$.

Para la evaluación del estado nutricional, se utilizó el método Índice de Masa Corporal $\left(\mathrm{IMC}=\mathrm{kg} / \mathrm{m}^{2}\right)$ con base en el peso y altura informados por los adultos mayores, preconizando los puntos de corte propuestos por la NSI (1992) (15).

Se recolectaron los datos socioeconómicos y de salud de los turistas de la tercera edad por medio de la aplicación de un cuestionario estructurado.

En la formación del banco de datos se utilizó el programa Microsoft Office Excel 2007.

Para el análisis de la asociación entre los promedios de consumo de energía, nutrientes y características de los adultos mayores, se optó por el Test-T no pareado, considerando significativas las diferencias con valor de $\mathrm{p}$ $<0,05$ (software GRAPHPAD INSTAD versión 3.01).

El Comité de Ética en Investigación de la UNIVALI (CAA-0197.0223.000.06) aprobó el proyecto y todos los 


\section{TABLA 1}

Perfil socioeconómico y de salud de los turistas de la tercera edad

\begin{tabular}{|c|c|c|}
\hline Variables & $\mathbf{N}$ & $\%$ \\
\hline \multicolumn{3}{|l|}{ Sexo } \\
\hline Femenino & 73 & 70 \\
\hline Masculino & 32 & 30 \\
\hline \multicolumn{3}{|l|}{ Edad } \\
\hline 60 a 74 años & 89 & 85 \\
\hline 75 años o más & 16 & 15 \\
\hline \multicolumn{3}{|l|}{ Nacionalidad } \\
\hline Argentina & 93 & 88 \\
\hline Brasileña y otras (Uruguaya, Paraguaya) & 12 & 12 \\
\hline \multicolumn{3}{|l|}{ Ejerce alguna profesión } \\
\hline Sí & 24 & 23 \\
\hline No & 81 & 77 \\
\hline \multicolumn{3}{|l|}{ Estado civil } \\
\hline Casado & 74 & 70 \\
\hline Otros & 31 & 30 \\
\hline \multicolumn{3}{|l|}{ Renta familiar } \\
\hline $\begin{array}{l}\text { 5,5 salarios mínimos } \\
(\$ 1080)\end{array}$ & 42 & 100 \\
\hline \multicolumn{3}{|l|}{ Escolaridad } \\
\hline Hasta 8 años & 23 & 23 \\
\hline De 9 a 11 años & 46 & 45 \\
\hline Más de 12 años & 33 & 32 \\
\hline \multicolumn{3}{|l|}{ Autopercepción de la salud } \\
\hline Excelente y buena & 98 & 93 \\
\hline Mala & 7 & 7 \\
\hline \multicolumn{3}{|l|}{ Hábito de fumar } \\
\hline Sí & 13 & 12 \\
\hline No & 92 & 88 \\
\hline \multicolumn{3}{|l|}{ Consumo de alcohol } \\
\hline No consumían & 69 & 66 \\
\hline Consumían ocasionalmente & 36 & 34 \\
\hline \multicolumn{3}{|l|}{ Práctica de actividad física } \\
\hline Tres o más veces por semana & 36 & 34 \\
\hline No practicaban & 69 & 66 \\
\hline \multicolumn{3}{|l|}{ Problemas con masticación } \\
\hline Sí & 13 & 12 \\
\hline No & 92 & 88 \\
\hline \multicolumn{3}{|l|}{ Pérdida de la palatabilidad } \\
\hline Sí & 2 & 2 \\
\hline No & 103 & 98 \\
\hline \multicolumn{3}{|l|}{ Pérdida del olfato } \\
\hline Sí & 6 & 6 \\
\hline No & 99 & 94 \\
\hline
\end{tabular}


sujetos llenaron un acuerdo de consentimiento libre y esclarecido para la investigación.

\section{RESULTADOS}

Los 105 turistas de la tercera edad que participaron en la investigación eran, en su mayoría, mujeres (70\%) procedentes de Argentina (88\%) con edad entre 60 y 74 anos $(85 \%)$, casados $(70 \%)$, con hasta 11 años de estudio formal (68\%). La renta familiar de los encuestados varió de \$ 172 a \$ 5347 dólares estadounidenses ${ }^{1}$ (tabla 1).

Una gran representación de los adultos mayores estudiados (93\%) dijo tener una buena o excelente salud, en cuanto a la ocurrencia de las siguientes enfermedades: hipertensión arterial sistémica por el $36 \%$, seguida de hipercolesterolemia por el $21 \%$, diabetes tipo 2 por el $13 \%$, alergias por el $9 \%$ e hipertrigliceridemia por el $8 \%$. En cuanto al uso de medicaciones, los más mencionados fueron los destinados al control de la hipertensión arterial, diabetes tipo 2 e hipercolesterolemia.

Con relación a los hábitos de vida, el $12 \%$ de los adultos mayores era fumador, el $34 \%$ consumía alcohol ocasionalmente y el $66 \%$ era sedentario, datos presentados en la tabla 1.

El IMC $\left(\mathrm{kg} / \mathrm{m}^{2}\right)$ de los individuos evaluados presentó un valor promedio de $25,43 \pm 8,20 \mathrm{~kg} / \mathrm{m}^{2}$ para hombres y de $24,12 \pm 8,88 \mathrm{~kg} / \mathrm{m}^{2}$ para mujeres. La clasificación del estado nutricional mostró una mayor ocurrencia de sobrepeso/obesidad entre el sexo masculino (57\%) cuando se comparó con el sexo femenino (42\%). El exceso de peso disminuyó con el aumento de la edad, alcanzando valores del $36 \%$ para los mayores de 75 años.

De los adultos mayores entrevistados, el 32\% dijo necesitar algún cuidado especial con la alimentación, destacándose, entre estos, el bajo consumo de alimentos ricos en grasa, relatado por el $28 \%$, seguido de la reducción de sal (26\%).

El análisis nutricional del consumo alimentario durante la cena presentó un porcentaje de adecuación excesivo para la mayoría de los nutrientes, estando dentro de las recomendaciones sólo para fibras (98\%) y carbohidratos $(102 \%)$, resultados presentes en la figura 1.

El estudio mostró una gran diferencia entre los valores medios de energía consumida $(1238,6$ cal) y las recomendaciones para la cena $(707 \mathrm{cal})$. La distribución porcentual media de los macronutrientes en la comida presentó valores recomendados sólo para las proteínas $(14,6 \%)$.

El porcentaje medio de calorías provenientes de los carbohidratos fue insuficiente $(43,6 \%)$, mientras que los valores de energía provista por la grasa total se presentaron elevados $(40,2 \%)$, siendo que el $11,8 \%$ de la grasa consumida en la comida era saturada.

Se observó que el consumo medio de colesterol (181,7 mg) estuvo por encima del límite máximo recomendado. La ingestión de sodio $(1323 \mathrm{mg})$ se mostró excesiva cuando se compara al $40 \%$ de la UL para este micronutriente (tabla 2).

\section{FIGURA 1}

Adecuación de energía y nutrientes consumidos (\%) en la cena por turistas de la tercera edad

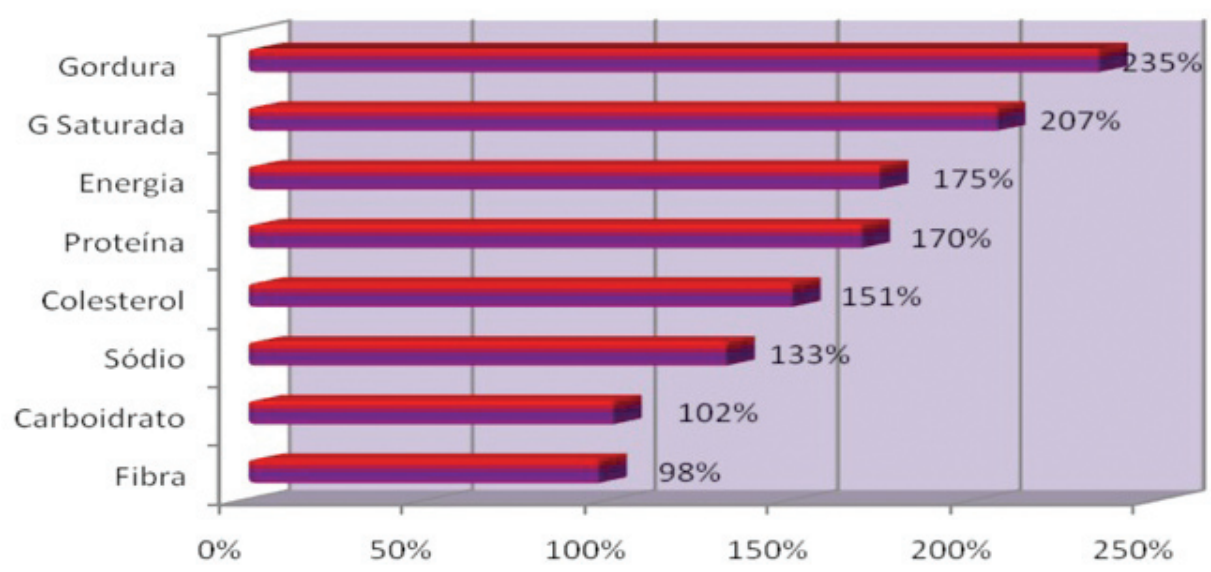

1 Valor del dólar $=1,80(10 / 10 / 07)$ 
La tabla 3 indica que no se encontraron diferencias entre las medias de consumo de energía y nutrientes por grupo etario o estado nutricional de los adultos mayores evaluados.

Los datos de consumo de grasa saturada mostraron diferencias estadísticamente significativas entre hombres y mujeres $(p=0,048)$. Se percibió una tendencia al mayor consumo de colesterol por el sexo masculino $(\mathrm{p}=0,066)$.

\section{DISCUSIÓN}

El presente estudio investigó el comportamiento alimentario de los turistas de la tercera edad en viaje. Los resultados de la investigación se refieren a la evaluación de sólo una comida en una única muestra, no representando la totalidad de la variabilidad de la dieta de los individuos estudiados.

El análisis de los resultados de este estudio se limita a las condiciones en que se dio el consumo de los ali-

\section{TABLA 2}

Consumo de energía y nutrientes (media y D.E.) por turistas de la tercera edad.

\begin{tabular}{|c|c|c|}
\hline \multicolumn{2}{|c|}{ Recomendación } & Consumo \\
\hline Energía (cal) & 707 & $1238,6 \pm 511,7$ \\
\hline Carbohidratos $(\%)$ & 55 a 75 & $44,6 \pm 11,0$ \\
\hline Proteínas (\%) & 10 a 15 & $14,6 \pm 5,8$ \\
\hline Grasa total (\%) & 15 a 30 & $40,2 \pm 9,4$ \\
\hline Grasa saturada (\%) & $\leq 10$ & $11,8 \pm 4,7$ \\
\hline Colesterol (mg) & $\leq 120$ & $181,7 \pm 139,5$ \\
\hline Sodio (mg) & $\leq 920$ & $1323 \pm 748,1$ \\
\hline Fibra (g) & $\geq 8,4$ & $8,2 \pm 5,7$ \\
\hline
\end{tabular}

\section{TABLA 3}

Valores medios de energía y nutrientes consumidos por turistas de la tercera edad, de acuerdo al sexo, grupo etario y estado nutricional

\begin{tabular}{|c|c|c|c|c|c|c|c|c|}
\hline Variables & $\begin{array}{l}\text { Energía } \\
\text { cal }\end{array}$ & $\begin{array}{c}\text { Proteína } \\
\%\end{array}$ & $\begin{array}{c}\text { Carbohidratos } \\
\%\end{array}$ & $\begin{array}{c}\text { Grasa total } \\
\%\end{array}$ & $\begin{array}{c}\text { Grasa saturada } \\
\%\end{array}$ & $\begin{array}{c}\text { Colesterol } \\
\text { mg }\end{array}$ & $\begin{array}{c}\text { Sodio } \\
\text { mg }\end{array}$ & $\begin{array}{c}\text { Fibra } \\
\text { g }\end{array}$ \\
\hline \multicolumn{9}{|l|}{ Grupo etario ${ }^{1}$} \\
\hline 60 a 74 años & 1218,00 & 14,73 & 45,41 & 39,82 & 11,61 & 178,47 & 1349,90 & 8,33 \\
\hline 75 años o más & 1353,10 & 14,02 & 43,34 & 42,64 & 13,21 & 199,66 & 1173,00 & 7,48 \\
\hline Valor de $\mathrm{p}$ & 0,333 & 0,654 & 0,500 & 0,273 & 0,214 & 0,578 & 0,386 & 0,589 \\
\hline \multicolumn{9}{|l|}{ Estado nutricional $^{1}$} \\
\hline Eutrófico & 1260,60 & 15,03 & 45,44 & 39,46 & 11,99 & 171,00 & 1414,40 & 8,88 \\
\hline Sobrepeso/obesidad & 1218,60 & 14,24 & 44,77 & 40,98 & 11,72 & 191,42 & 1239,80 & 7,59 \\
\hline Valor de $\mathrm{p}$ & 0,676 & 0,487 & 0,761 & 0,412 & 0,769 & 0,456 & 0,234 & 0,478 \\
\hline \multicolumn{9}{|l|}{$\operatorname{Sexo}^{1}$} \\
\hline Masculino & 1316,70 & 13,73 & 45,21 & 41,05 & 13,11 & 216,34 & 1455,70 & 8,65 \\
\hline Femenino & 1197,90 & 14,69 & 45,60 & 39,83 & 11,19 & 163,62 & 1253,70 & 7,96 \\
\hline Valor de $\mathrm{p}$ & 0,261 & 0,368 & 0,857 & 0,531 & $0,048^{*}$ & 0,066 & 0,190 & 0,131 \\
\hline
\end{tabular}

(*) indica diferencias estadísticas significativas $(\mathrm{p}<0,05)$.

${ }^{1}$ Test $\mathrm{T}$ no pareado. 
mentos, ya que durante las vacaciones, probablemente los turistas de la tercera edad hayan elegido opciones alimentarias no usuales, influenciados por la gran variedad de alimentos ofrecidos por el buffet del restaurante y por la curiosidad en conocer nuevos sabores.

Por otra parte, la alta prevalencia de adultos mayores originarios de Argentina y otros países de Sudamérica también puede haber ejercido influencia sobre los resultados, considerándose que la cultura tiene un importante papel en el comportamiento alimentario (16).

El nivel socioeconómico de los turistas mayores que visitaban el Balneario Camboriú también puede haber interferido en los resultados. De acuerdo con la investigación realizada en este municipio en 329 turistas mayores utilizando el criterio ABA-ABIPEME de clasificación social (17), el $47 \%$ fue clasificado como pertenecientes a la clase C, seguidos del $28 \%$ de la clase B y del $19 \%$ da clase D. A su vez, los turistas pertenecientes a las clases A y E sumaron solamente el 6\% de la muestra (10).

Cabe destacar que esta muestra no es representativa para la población de mayores en general, pues el estudio fue realizado con personas en viaje de turismo. Es probable que estos individuos hayan presentado mejores condiciones físicas y psicológicas que otros individuos del mismo grupo etario.

Aún relatando una alta prevalencia de enfermedades características de esta fase de la vida, la mayoría de los adultos mayores estudiados (93\%) presentó una óptima o buena percepción de su salud, probablemente a consecuencia de poseer un mayor grado de independencia física, y de su propia condición de turista, oportunidad en la que las personas acostumbran presentar mayor relajación y tranquilidad (18).

La excelente auto percepción de la salud referida en este trabajo es una característica encontrada también en otros estudios con turistas, y que refleja una realidad un poco diferente de otras investigaciones realizadas con personas mayores $(19,20)$.

En el 2006, un trabajo realizado con turistas mayores hospedados en hoteles de Balneário Camboriú verificó que el $94 \%$ de los entrevistados consideraba su salud buena o excelente (10). Datos semejantes fueron identificados por Pestana y Gageiro (21) en un estudio también realizado con turistas seniors en viaje por Europa. En la referida investigación, de los 465 individuos entrevistados, el $79 \%$ consideró su salud buena o muy buena.

Algunos estudios asocian la auto percepción positiva al grado de independencia física $(19,20)$, a la condición socioeconómica $(20,22)$ y al mayor relajamiento y tranquilidad durante las vacaciones (10).

La percepción favorable de la propia salud, juntamente con la buena condición oral y sensorial presentada por la mayoría de los entrevistados, puede haber influido en el consumo alimentario evaluado.

Aunque el $12 \%$ de los adultos mayores mencionó dificultades de masticación, la mayoría de ellos no presentó problemas en la cavidad oral o pérdida de la palatabilidad de los alimentos, datos que se diferencian de los encontrados en la literatura para este grupo de edad (23).

En los últimos veinte años, tanto Brasil como otros países de América Latina están pasando por una rápida transición demográfica, epidemiológica y nutricional, destacándose el aumento de la obesidad en los diversos grupos de población (24).

Se observó, en el presente estudio, que pese a que el IMC medio estaba en los valores normales para este grupo etario, el $42 \%$ de los adultos mayores presentó exceso de peso. Sin embargo, no se encontró una relación positiva entre el consumo de energía de la comida y el estado nutricional $(\mathrm{p}=0,67)$.

Es posible que parte de la inconsistencia entre el estado nutricional y la ingestión calórica en la comida pueda explicarse por la reducción de las necesidades de energía como consecuencia del envejecimiento, junto a la disminución de actividad física. Cabe destacar también que mientras el estado nutricional es resultado de la historia alimentaria de los individuos, la presente investigación se limitó a la evaluación del consumo de una sola comida.

La participación de la gran mayoría de los adultos mayores con edades entre 60 y 74 años, juntamente con una escolaridad por encima de 9 años puede haber influenciado los índices de exceso de peso encontrados.

Según Campos et al., (25) la asociación del sobrepeso con el grupo etario indica que los adultos mayores de más edad poseen una probabilidad menor de presentar sobrepeso y obesidad, y que los adultos mayores con 9 a 11 años de escolaridad tienen 2,36 veces mayor probabilidad de poseer sobrepeso cuando se comparan a adultos mayores con 4 años o menos de escolaridad.

Algunos estudios indican el proceso de globalización y el consumo de alimentos industrializados, incorporado al estilo de vida sedentario y al alto consumo de alimentos ricos en lípidos, azúcar y pobre en fibras, como responsable por los altos índices de exceso de peso asociado a enfermedades crónicas, como las enfermedades cardiovasculares y diabetes (26).

América Latina utiliza como modelo el estándar alimentario estadounidense, rico en grasas y azúcar refinado. Esta dieta, pobre en carbohidratos complejos (fuente importante de fibra alimentaria), tiene su mayor consumo centrado en las proteínas de origen animal.

Los estudios muestran también que en este estándar 
alimentario, la dieta es rica en sal, al estar constituída por alimentos altamente industrializados (27).

La adopción de la dieta "afluente" está expandiéndose, sobre todo en situaciones de prosperidad económica (28).

El análisis del consumo de alimentos durante la cena de los turistas de la tercera edad refleja, además del consumo elevado de energía, el reducido tenor de carbohidratos complejos y el exceso de grasas, con destacando las grasas saturadas y el colesterol.

Algunos autores indican este tipo de dieta como factor de riesgo para enfermedades crónicas no transmisibles tales como obesidad, hipertensión arterial, enfermedades cardiovasculares, cáncer y diabetes tipo 2 (29).

La correlación entre la cantidad de grasas y el sexo mostró resultados semejantes a otros estudios, en que el consumo de grasas saturadas y colesterol fue mayor entre la población masculina, lo que sugiere una tendencia de las mujeres a elegir sus alimentos de manera más sana $(30,31)$.

Se notó en la presente investigación un elevado consumo de sal durante la cena con adecuación equivalente al 147\% del nivel máximo de ingestión tolerable.

Estudios recientes evidencian que una dieta baja en sodio ayuda a mantener o disminuir la presión arterial, cuando se asocia a una alimentación pobre en ácidos grasos saturados y alta en carbohidratos (29).

Pese al hecho que sólo el $36 \%$ de los entrevistados relató hipertensión arterial, algunos estudios muestran prevalencia del desconocimiento de la condición de hipertenso entre los adultos mayores que varían del 23,4\% al 50,0\%, siendo importante el control de las cantidades de este nutriente en la dieta $(32,33)$.

La práctica alimentaria entre turistas de la tercera edad mostró una elevada inadecuación de consumo durante la cena. Se sabe que una dieta desequilibrada en nutrientes es un factor de riesgo para innumerables enfermedades, en especial en esta fase de la vida, debido a los cambios fisiológicos provenientes del aumento de la edad.

Con el envejecimiento, la adecuación de una dieta rica en nutrientes se hace más importante, ya que los alimentos, además de promover salud, pueden auxiliar en el tratamiento y prevención de las enfermedades crónicas no transmisibles, muy comunes en este grupo etario.

Los resultados encontrados indican la necesidad de que el restaurante del hotel ofrezca en sus servicios orientaciones sobre prácticas alimentarias sanas dirigidas a los adultos mayores, y así ser una alternativa para la tercera edad en la promoción de hábitos alimentarios adecuados.
Se destaca la escasez de estudios sobre el consumo alimentario de turistas de la tercera edad, registrándose la necesidad de nuevas investigaciones por parte de estudiantes de graduación y profesionales del área de la salud.

\section{RESUMEN}

Objetivo: evaluar la calidad nutricional de la cena consumida por 105 turistas de la tercera edad hospedados en un hotel. Método: para la estandarización previa del peso medio de los alimentos consumidos fueron pesadas tres porciones de cada una de las opciones ofrecidas. El consumo alimentario fue verificado por la observación directa de las porciones servidas en el plato, convertidas posteriormente en gramos. En la evaluación de la composición nutricional de la comida, se consideraron los datos sobre valor energético, macronutrientes, grasa saturada, colesterol, sodio y fibra. Resultados: la mayoría de los turistas era de Argentina (88\%), del sexo femenino $(70 \%)$, con edades entre 60 y 84 años $(85 \%)$. Se notó un alto consumo de energía (175\%), grasas totales (235\%), grasas saturadas (207\%), colesterol $(151 \%)$, proteína (170\%) y sodio (133\%). Se observó un consumo mayor de grasa saturada entre el sexo masculino. La ingestión fue adecuada para carbohidratos (102\%) y fibras (98\%).

Palabras clave: Adulto mayor; turismo; dietética; consumo de alimentos; hotel.

Dirigir la correspondencia a:

Profesora

Marcia Reis F.

Centro de Ciencias de la Salud

Universidad del Valle del Itajaí

Santa Catarina

Brasil

\section{BIBLIOGRAGÍA}

1. Noronha KVM de S. Andrade MV. Social inequality in health and the utilization of health services among the elderly in Latin America. Rev Panam Salud Pública 2005; 17: 410-418.

2. Filho-Coelho JM. Ramos LR. Epidemiologia do envelhecimento no Nordeste do Brasil: resultados de inquérito alimentar. Rev Saúde Pública 1999; 33: 445-453.

3. Camarano AA. Pasinato MT. Envelhecimento, pobreza e proteção social na América Latina. II Congreso de la Asociación Latinoamericana de Población. Guadalajara (México), pp 2-17, 2006.

4. Ferrioli E. et al. Aspectos do metabolismo energético e protéico dos idosos. Rev Nutrire 2000; 
19/20: 19-30.

5. Cervi A. Franceschini S do CC. Priore SE. Análise crítica do uso do índice de massa corporal para idosos. Rev Nutr 2005; 18: 765-775.

6. Monteiro C.S. A influência da nutrição no bem-estar de idosas. Nutr Pauta 2004; 17: 26-29.

7. Campos MTF de S. Monteiro JBR. Ornelas APR de C. Fatores que afetam o consumo alimentar e a nutrição do idoso. Rev Nutr 2000; 13: 157-165.

8. Fagundes RLM. Cunha AC de. Avaliação do cardápio e sua implicação no estado nutricional em idosos. Nutr Pauta 2004; 2: 38-43.

9. Fromer B. Vieira DD. Turismo e terceira idade. Aleph, São Paulo, 2003.

10. Felipe MR. Atenção alimentar e nutricional a turistas idosos: um estudo da rede hoteleira de Balneário Camboriú/SC. Balneário Camboriú: UNIVALI/ Programa de Doutorado em Turismo e Hotelaria, 2006.

11. Food and Nutrition Board. Institute of Medicine. Dietary reference intakes for energy, carbohydrate, fiber, fat, fatty, acids, cholesterol, protein, and amino acids. National Academy Press, Washington, 2002; 1-2.

12. Sá, NG. Nutrição e dietética. 7 ed. Nobel, São Paulo, 1990.

13. FAO. Joint WHO/FAO Expert Consultation on Diet, Nutrition and the Prevention of Chronic Diseases, Geneva, 2003.

14. Food and Nutrition Board. Institute of Medicine. Dietary reference intakes for water, potassium, sodium, chloride, and sulfate. National Academy Press, Washington, 2004; 450.

15. Nutrition Screening Initiative. Interventions manual for professionals caring for older Americans. Washington, DC, 1992.

16. Garcia RWD. Reflexos da globalização na cultura alimentar: considerações sobre as mudanças na alimentação urbana. Rev Nutr 2003; 16: 483-492.

17. Denker AFM. Métodos e técnicas de pesquisa em turismo. Futura, São Paulo, 1998.

18. Gaspari JC. Schwartz G. The elderly and the emotional resignification of leisure. Psic Teor e Pesq 2005; 21:69-76.

19. Lebrão ML. Laurenti R. Saúde, bem-estar e envelhecimento: o estudo SABE no Município de São Paulo. Rev Bras Epidemiol. 2005;8:127-141.

20. Alves LS. Rodrigues RN. Determinantes da autopercepção de saúde entre idosos do município de São
Paulo. Rev Panam Salud Pública 2005;17:333-341.

21. Pestana H. Gageiro J. Turismo e envelhecimento demográfico: uma realidade de futuro. www.apdemografia.pt/pdf_congresso/5Helena_Pestana_e_ João_Gageiro.pdf.

22. Rosa TE da C. Benício MH d A. Latorre M do RD de OL. Ramos LR. Fatores determinantes da capacidade funcional entre idosos. Rev Saúde Pública 2003; 37:40-48.

23. Silva SRC. Valsecki A J. Avaliação das condições de saúde dos idosos em um município brasileiro. Rev Panam Salud Pública 2000; 8: 268-271.

24. Kac G. Velásquez-Meléndez G. A transição nutricional e a epidemiologia da obesidade na América Latina. Cad Saúde Pública 2003; 19: S4-S5.

25. Campos MAG. Pedroso ERP. Lamounier JA. Colosimo EA. Abrantes MM. Estado nutricional e fatores associados em idosos. Rev Assoc Med Bras 2006; 52: 214-221.

26. Barría PRP. Amigo HC. Transición nutricional: una revisión del perfil latinoamericano. Arch Latinoam Nutr 2006; 56: 03-11.

27. Tagle MA. Cambios en los patrones de consumo alimentario en América Latina. Arch Latinoam Nutr 1988; 38: 750-765.

28. Popkin BM. Urbanization, lifestyle changes and the nutrition transition. World Dev 1999; 27: 19501916.

29. Restrepo SLM. Morales RMG. Ramírez MCG. López MVL. Varela LEL. Los hábitos alimentarios en el adulto mayor y su relación con los procesos protectores y deteriorentes en salud. Rev Chil Nutr 2006; 33: 500-510.

30. Lopes ACS. Caiaffa WT. Sichieri R. Mingoti SA. Lima-Costa MF. Consumo de nutrientes em adultos e idosos em estudo de base populacional: Projeto Bambuí. Cad Saúde Pública 2005; 21: 1201-1209.

31. Cesar TB. Wada SR. Borges RG. Zinco plasmático e estado nutricional em idosos. Rev Nutr 2005; 18: 357-365.

32. Firmo JOA. Uchôa E. Lima-Costa MF. Projeto Bambuí: fatores associados ao conhecimento da condição de hipertenso entre idosos. Cad Saúde Pública 2004; 20: 1029-1040.

33. Burstyn M. Shpilberg O. Ginsberg GM. Cohen A. Stessanan J. Hypertension in the Jerusalem 70 year olds study population: prevalence, awareness, treatment and control. Isr J Med Sci 1996; 36: 629-330. 